Supporting Information

\title{
Synthesis, Structures, and Photoluminescent Properties of Tricyanidonitridorhenium(V) Complexes with Bipyridine-Type Ligands
}

Kojiro Nagata, ${ }^{\dagger}$ Naoko Otsuji,,${ }^{\ddagger}$ Soichiro Akagi, ${ }^{\S}$ Sho Fujii, ${ }^{\S}$ Noboru Kitamura, ${ }^{\S}$ and Takashi Yoshimura*,

Radioisotope Research Center, Institute for Radiation Sciences, Osaka University, Suita 5650871, Japan, "Department of Chemistry, Graduate School of Science, Osaka University, Toyonaka 560-0043, Japan, ${ }^{\circledR}$ Department of Chemical Sciences and Engineering, Graduate School of Chemical Sciences and Engineering, Hokkaido University, Sapporo 060-0810, Japan, "Project Research Center for Fundamental Sciences, Graduate School of Science, Osaka University, Toyonaka 560-0043, Japan 
Table S1. Crystallographic Data of $\mathbf{R e}-\mathbf{X}_{2} \mathbf{b p y}\left(\mathrm{X}=\mathrm{NMe}_{2}, \mathrm{NH}_{2}, \mathrm{OMe}, \mathrm{Me}, \mathrm{H}, \mathrm{Cl}, \mathrm{Br}\right)$

\begin{tabular}{|c|c|c|c|c|c|c|c|}
\hline Complex & $\begin{array}{c}\mathrm{Re}- \\
\left(\mathrm{NMe}_{2}\right)_{2} \mathbf{b p y}\end{array}$ & $\begin{array}{c}\text { Re- } \\
\left(\mathrm{NH}_{2}\right)_{2} \text { bpy }\end{array}$ & $\begin{array}{c}\text { Re- } \\
(\mathrm{OMe})_{2} \text { bpy }\end{array}$ & Re-Me ${ }_{2} b p y$ & Rebpy & $\mathrm{Re}-\mathrm{Cl}_{2} \mathrm{bpy}$ & $\operatorname{Re}-\mathrm{Br}_{2} \mathbf{b p y}$ \\
\hline Formula & $\mathrm{C}_{45} \mathrm{H}_{48} \mathrm{~N}_{10} \mathrm{O}_{2} \mathrm{PRe}$ & $\mathrm{C}_{37} \mathrm{H}_{30} \mathrm{~N}_{8} \mathrm{PRe}$ & $\mathrm{C}_{39} \mathrm{H}_{32} \mathrm{~N}_{6} \mathrm{O}_{2} \mathrm{PRe}$ & $\mathrm{C}_{39} \mathrm{H}_{32} \mathrm{~N}_{6} \mathrm{PRe}$ & $\mathrm{C}_{37} \mathrm{H}_{28} \mathrm{~N}_{6} \mathrm{PRe}$ & $\mathrm{C}_{37} \mathrm{H}_{26} \mathrm{Cl}_{2} \mathrm{~N}_{6} \mathrm{PRe}$ & $\mathrm{C}_{37} \mathrm{H}_{26} \mathrm{Br}_{2} \mathrm{~N}_{6} \mathrm{PRe}$ \\
\hline $\mathrm{Fw}$ & 978.11 & 803.87 & 833.89 & 801.89 & 773.83 & 842.72 & 931.62 \\
\hline $\begin{array}{l}\text { Crystal } \\
\text { System }\end{array}$ & triclinic & orthorhombic & triclinic & monoclinic & monoclinic & monoclinic & monoclinic \\
\hline $\begin{array}{l}\text { Space } \\
\text { Group }\end{array}$ & $P \overline{1}$ & $P$ bca & $P \overline{1}$ & $P 2_{1} / n$ & $P 2_{1} / c$ & $P 2{ }_{1} / a$ & $P 2_{1} / a$ \\
\hline$a / \AA ̊$ & $9.7387(2)$ & $28.112(2)$ & $11.4291(5)$ & $9.8634(3)$ & $14.5389(4)$ & $14.3082(3)$ & $14.2650(3)$ \\
\hline$b / \AA ̊$ & $11.3508(2)$ & $16.6561(9)$ & $12.5935(5)$ & $20.4567(6)$ & $14.2353(3)$ & $15.2636(4)$ & $15.3046(3)$ \\
\hline$c / \AA ̊$ & $20.6184(4)$ & $16.1027(9)$ & $12.7761(9)$ & $16.8056(5)$ & $15.5838(4)$ & $16.0535(4)$ & $16.1371(3)$ \\
\hline$\alpha / \operatorname{deg}$ & $91.532(6)$ & & $82.341(6)$ & & & & \\
\hline$\beta / \operatorname{deg}$ & $100.056(7)$ & & $79.403(6)$ & $93.079(7)$ & $91.169(6)$ & $103.140(7)$ & $102.866(7)$ \\
\hline$\gamma / \operatorname{deg}$ & $95.076(7)$ & & $70.184(5)$ & & & & \\
\hline$V / \AA^{3}$ & 2233.32(9) & $7539.8(8)$ & $1695.50(17)$ & $3386.02(18)$ & $3224.63(14)$ & $3414.20(17)$ & $3434.60(15)$ \\
\hline$Z$ & 2 & 8 & 2 & 4 & 4 & 4 & 4 \\
\hline$\rho_{\text {calc }} / \mathrm{gcm}^{-3}$ & 1.454 & 1.416 & 1.633 & 1.573 & 1.594 & 1.640 & 1.802 \\
\hline$\mu / \mathrm{mm}^{-1}$ & 2.805 & 3.301 & 3.676 & 3.673 & 3.854 & 3.799 & 5.948 \\
\hline$R_{1}$ & 0.0657 & 0.0804 & 0.0519 & 0.0424 & 0.0502 & 0.0911 & 0.0928 \\
\hline $\mathrm{w} R_{2}$ & 0.1498 & 0.1841 & 0.1122 & 0.0673 & 0.1061 & 0.1989 & 0.1775 \\
\hline GOF & 1.327 & 1.074 & 1.293 & 1.028 & 1.346 & 1.353 & 1.416 \\
\hline
\end{tabular}


Table S2. Atomic Coordinates of Optimized Geometry of Complex Anion of Re-(NMe $\mathbf{N}_{2} \mathbf{b p y}$

\begin{tabular}{|c|c|c|c|}
\hline Atom & $x / \AA$ & $y / \AA$ & $z / \AA$ \\
\hline Re1 & -1.40014850 & 7.94320702 & 13.38212223 \\
\hline N1 & -0.75137383 & 9.48702326 & 13.28090731 \\
\hline $\mathrm{N} 2$ & -4.01342551 & 8.15098765 & 11.40186767 \\
\hline N3 & -3.42315086 & 7.95424017 & 15.92083443 \\
\hline N4 & 0.80782047 & 6.38380290 & 15.24420056 \\
\hline N5 & -0.35682182 & 7.09481968 & 11.70869367 \\
\hline N6 & -2.03583184 & 5.60447040 & 13.10059559 \\
\hline N7 & 1.84233233 & 5.56525759 & 8.39671077 \\
\hline N8 & -2.88543763 & 1.55944505 & 12.35508056 \\
\hline $\mathrm{C} 1$ & -3.10175080 & 8.12765142 & 12.14348311 \\
\hline $\mathrm{C} 2$ & -2.67420812 & 8.04737110 & 15.01902540 \\
\hline $\mathrm{C} 3$ & 0.00638608 & 6.98805853 & 14.63276468 \\
\hline $\mathrm{C} 4$ & 0.50812105 & 7.86398418 & 10.98592200 \\
\hline $\mathrm{C} 5$ & 1.22414265 & 7.41550910 & 9.89862451 \\
\hline C6 & 1.09539879 & 6.07141293 & 9.46429559 \\
\hline $\mathrm{C} 7$ & 0.19681186 & 5.28302729 & 10.19212619 \\
\hline $\mathrm{C} 8$ & -0.50228920 & 5.79113721 & 11.29537862 \\
\hline C9 & -2.91506729 & 4.93601780 & 13.85933533 \\
\hline $\mathrm{C} 10$ & -3.24204002 & 3.60642256 & 13.64678895 \\
\hline $\mathrm{C} 11$ & -2.62063302 & 2.90239680 & 12.58697708 \\
\hline $\mathrm{C} 12$ & -1.71370916 & 3.62170385 & 11.78636531 \\
\hline $\mathrm{C} 13$ & -1.43860767 & 4.96554862 & 12.07188348 \\
\hline $\mathrm{C} 14$ & 2.38977871 & 6.51286010 & 7.43498522 \\
\hline $\mathrm{C} 15$ & 1.45382815 & 4.27445499 & 7.85194328 \\
\hline $\mathrm{C} 16$ & -2.43520657 & 0.96255865 & 11.10868339 \\
\hline $\mathrm{C} 17$ & -4.03374286 & 0.94923375 & 13.00937010 \\
\hline $\mathrm{H} 1$ & 0.59923251 & 8.89266214 & 11.33208965 \\
\hline $\mathrm{H} 2$ & 1.88001922 & 8.12367744 & 9.39700620 \\
\hline $\mathrm{H} 3$ & 0.02401745 & 4.24849326 & 9.91063513 \\
\hline $\mathrm{H} 4$ & -3.37030481 & 5.51363091 & 14.66788588 \\
\hline H5 & -3.97271190 & 3.13658491 & 14.30088168 \\
\hline H6 & -1.22544402 & 3.13946870 & 10.94442022 \\
\hline $\mathrm{H} 7$ & 2.98016900 & 5.96009437 & 6.69377985 \\
\hline H8 & 1.60729537 & 7.09041648 & 6.90417737 \\
\hline H9 & 3.06509273 & 7.22252274 & 7.93048412 \\
\hline $\mathrm{H} 10$ & 2.15643953 & 4.00011840 & 7.055544447 \\
\hline H11 & 1.51033848 & 3.49805169 & 8.62829896 \\
\hline $\mathrm{H} 12$ & 0.42681354 & 4.27167471 & 7.43293640 \\
\hline $\mathrm{H} 13$ & -2.70884220 & -0.09900437 & 11.10467151 \\
\hline H14 & -2.88028208 & 1.44527130 & 10.21681657 \\
\hline H15 & -1.34017542 & 1.02441209 & 11.02159385 \\
\hline H16 & -4.06167025 & -0.11631656 & 12.75293181 \\
\hline H17 & -3.94225875 & 1.02647497 & 14.10176857 \\
\hline H18 & -4.99347633 & 1.41130412 & 12.70941616 \\
\hline
\end{tabular}


Table S3. Atomic Coordinates of Optimized Geometry of Complex Anion of Re-(OMe) 2 bpy

\begin{tabular}{llll}
\hline Atom & $x / \AA$ & $y / \AA$ & $z / \AA$ \\
\hline Re1 & 7.05015262 & 14.18462905 & 2.24085153 \\
O1 & 6.63456073 & 11.65580999 & -3.55970436 \\
O2 & 4.21947893 & 8.23499167 & 2.40705851 \\
N1 & 7.78403286 & 15.61468471 & 1.76118952 \\
N2 & 3.86105319 & 14.88877309 & 1.95066785 \\
N3 & 6.71499655 & 14.36640716 & 5.46197407 \\
N4 & 9.61511045 & 12.20102471 & 2.72615785 \\
N5 & 6.89886507 & 13.30570333 & 0.29454282 \\
N6 & 5.99632795 & 11.98014277 & 2.40389781 \\
C1 & 5.00939607 & 14.68988630 & 2.09958113 \\
C2 & 6.87014947 & 14.38964047 & 4.29714618 \\
C3 & 8.72241511 & 12.95424801 & 2.59913603 \\
C4 & 7.36487027 & 13.98676199 & -0.78819675 \\
C5 & 7.31461047 & 13.51362952 & -2.08584946 \\
C6 & 6.74957255 & 12.24871595 & -2.32505470 \\
C7 & 6.26748569 & 11.53521368 & -1.23542359 \\
C8 & 6.34493831 & 12.06496219 & 0.05674063 \\
C9 & 5.57263918 & 11.39671621 & 3.53702172 \\
C10 & 4.97785717 & 10.14606236 & 3.55681586 \\
C11 & 4.81388390 & 9.46698033 & 2.33769338 \\
C12 & 5.25163683 & 10.06572766 & 1.15839982 \\
C13 & 5.84658631 & 11.33944232 & 1.22964641 \\
C14 & 7.13524562 & 12.40195684 & -4.66956500 \\
C15 & 4.04279573 & 7.53572005 & 1.17371005 \\
H1 & 7.79459386 & 14.96137864 & -0.56137580 \\
H2 & 7.71568697 & 14.13828924 & -2.88143113 \\
H3 & 5.82795710 & 10.55434495 & -1.40740186 \\
H4 & 5.72779427 & 11.97652422 & 4.45015738 \\
H5 & 4.64149587 & 9.69268184 & 4.48865254 \\
H6 & 5.13866798 & 9.56565420 & 0.20061472 \\
H7 & 8.21545191 & 12.59697486 & -4.56841481 \\
H8 & 6.95888053 & 11.77824879 & -5.55290974 \\
H9 & 6.60088454 & 13.35924807 & -4.78431935 \\
H10 & 3.55736340 & 6.58916181 & 1.43502932 \\
H11 & 3.39750090 & 8.10138697 & 0.48201943 \\
H12 & 5.01055475 & 7.33082459 & 0.68759860 \\
\hline & & & \\
\hline & & & \\
\hline
\end{tabular}


Table S4. Atomic Coordinates of Optimized Geometry of Complex Anion of Rebpy

\begin{tabular}{llll}
\hline Atom & $x / \AA$ & $y / \AA$ & $z / \AA$ \\
\hline Re1 & 1.64308305 & 3.18502102 & 5.74306314 \\
N1 & 1.70737666 & 4.03459584 & 7.19262607 \\
N2 & 4.82249520 & 2.51858552 & 5.32804122 \\
N3 & 1.94069912 & 5.59167950 & 3.58914423 \\
N4 & -1.58633941 & 3.20296299 & 5.19377045 \\
N5 & 1.40961431 & 1.18225490 & 6.58522002 \\
N6 & 1.51348995 & 1.56744040 & 3.93744969 \\
C1 & 3.68406610 & 2.77269837 & 5.47784007 \\
C2 & 1.83226519 & 4.73784715 & 4.39217773 \\
C3 & -0.42757218 & 3.20845454 & 5.39424484 \\
C4 & 1.36211798 & 1.01792204 & 7.93390765 \\
C5 & 1.21455669 & -0.22136745 & 8.53771082 \\
C6 & 1.11035442 & -1.35961736 & 7.73617262 \\
C7 & 1.15651107 & -1.20377846 & 6.35712818 \\
C8 & 1.30470798 & 0.06968847 & 5.79347730 \\
C9 & 1.57429844 & 1.85768470 & 2.62671128 \\
C10 & 1.47673799 & 0.87786575 & 1.64316052 \\
C11 & 1.30634713 & -0.44964264 & 2.04127775 \\
C12 & 1.24425688 & -0.75250889 & 3.39809876 \\
C13 & 1.35405881 & 0.28375791 & 4.33579211 \\
H1 & 1.44603135 & 1.92870947 & 8.52321972 \\
H2 & 1.18296631 & -0.28127459 & 9.62523874 \\
H3 & 0.99374653 & -2.34976687 & 8.17683654 \\
H4 & 1.07820575 & -2.07406053 & 5.70904872 \\
H5 & 1.70411322 & 2.91094729 & 2.37348012 \\
H6 & 1.53227512 & 1.15552579 & 0.59098120 \\
H7 & 1.22214710 & -1.24547277 & 1.30065025 \\
H8 & 1.11050466 & -1.78362398 & 3.71849406 \\
\hline
\end{tabular}


Table S5. Atomic Coordinates of Optimized Geometry of Complex Anion of Re-Br 2 bpy

\begin{tabular}{llll}
\hline Atom & $x / \AA$ & $y / \AA$ & $z / \AA$ \\
\hline Re1 & 0.88210384 & 4.24209145 & 7.08992521 \\
Br1 & -0.21838642 & 9.18903567 & 2.49041768 \\
Br2 & -0.25089015 & 10.51931258 & 10.22677862 \\
N1 & 1.07402292 & 3.00786747 & 5.97313085 \\
N2 & 3.90342324 & 5.49338142 & 7.35069044 \\
N3 & 1.29913495 & 2.94845265 & 10.03869524 \\
N4 & -2.34706008 & 4.21836541 & 7.66378107 \\
N5 & 0.51695300 & 5.80999529 & 5.70617803 \\
N6 & 0.51849223 & 6.33493973 & 8.31693653 \\
C1 & 2.85365323 & 4.97182390 & 7.27760445 \\
C2 & 1.16310915 & 3.31474313 & 8.93061951 \\
C3 & -1.18945711 & 4.15411152 & 7.47543385 \\
C4 & 0.52166182 & 5.54459367 & 4.36230820 \\
C5 & 0.31215297 & 6.49820369 & 3.38854303 \\
C6 & 0.07850058 & 7.81909064 & 3.79379960 \\
C7 & 0.06338176 & 8.12991733 & 5.13708611 \\
C8 & 0.28352458 & 7.11865777 & 6.08450538 \\
C9 & 0.53523728 & 6.47726744 & 9.64830297 \\
C10 & 0.31248804 & 7.70134343 & 10.27281020 \\
C11 & 0.06413714 & 8.79688215 & 9.44478753 \\
C12 & 0.04145807 & 8.67301395 & 8.06681458 \\
C13 & 0.27808094 & 7.39747709 & 7.51992569 \\
H1 & 0.70862180 & 4.50312167 & 4.10363905 \\
H2 & 0.33194786 & 6.21621899 & 2.33660248 \\
H3 & -0.11643408 & 9.15309199 & 5.46048666 \\
H4 & 0.73779846 & 5.56623152 & 10.21890084 \\
H5 & 0.33185385 & 7.79228805 & 11.35766768 \\
H6 & -0.15623048 & 9.54156971 & 7.44187890 \\
\hline & & &
\end{tabular}


Table S6. Calculated Selected Bond Distances $(\AA)$ and Angles (deg) of Complex Anions of Re$\mathbf{X}_{2}$ bpy $\left(\mathrm{X}=\mathrm{NMe}_{2}, \mathrm{OMe}, \mathrm{H}\right.$, and $\left.\mathrm{Br}\right)$

\begin{tabular}{ccccc}
\hline Complex & $\begin{array}{c}\text { Re- } \\
(\mathbf{N M e})_{2} \mathbf{b p y}\end{array}$ & $\begin{array}{c}\text { Re- } \\
(\mathbf{O M e})_{2} \mathbf{b p y}\end{array}$ & Rebpy & Re-Br2bpy \\
\hline $\mathrm{B} e=\mathrm{N}$ & 1.6777 & 1.6774 & 1.6814 & 1.6755 \\
$\mathrm{Re}-\mathrm{C} 1$ & 2.1127 & 2.1071 & 2.0990 & 2.1106 \\
$\mathrm{Re}-\mathrm{C} 2$ & 2.0769 & 2.0743 & 2.0669 & 2.0802 \\
$\mathrm{Re}-\mathrm{C} 3$ & 2.1106 & 2.1068 & 2.1000 & 2.1090 \\
$\mathrm{Re}-\mathrm{N}(e q)$ & 2.1468 & 2.1409 & 2.1851 & 2.1228 \\
$\mathrm{Re}-\mathrm{N}(a x)$ & 2.4399 & 2.4489 & 2.4277 & 2.4531 \\
\hline & & Bond Angle/deg & & \\
\hline $\mathrm{N} \equiv \mathrm{Re}-\mathrm{C} 1$ & 101.29 & 101.54 & 99.85 & 101.94 \\
$\mathrm{~N} \equiv \mathrm{Re}-\mathrm{C} 2$ & 103.81 & 103.72 & 100.39 & 104.24 \\
$\mathrm{~N} \equiv \mathrm{Re}-\mathrm{C} 3$ & 101.21 & 101.49 & 100.09 & 101.75 \\
$\mathrm{~N} \equiv \mathrm{Re}-\mathrm{N}(e q)$ & 97.39 & 96.95 & 97.75 & 97.43 \\
$\mathrm{~N} \equiv \mathrm{Re}-\mathrm{N}(a x)$ & 167.39 & 167.10 & 168.50 & 168.18 \\
\hline & & & & \\
\hline
\end{tabular}


Table S7. Calculated Energies and Components of MOs Near Frontier Orbital Levels for Complex Anion of Re-(NMe $)_{2} \mathbf{b p y}$

\begin{tabular}{|c|c|c|c|c|c|}
\hline & Energy / eV & $\mathrm{Re}$ & $\mathrm{N}$ & $\mathrm{CN}$ & $\left(\mathrm{NMe}_{2}\right)_{2} \mathrm{bpy}$ \\
\hline LUMO+6 & 0.26 & 0.13 & 0.05 & 0.22 & 0.60 \\
\hline LUMO+5 & 0.16 & 0.07 & & 0.09 & 0.85 \\
\hline LUMO+4 & -0.61 & 0.09 & 0.09 & & 0.82 \\
\hline LUMO+3 & -1.02 & 0.12 & 0.11 & 0.01 & 0.76 \\
\hline $\mathrm{LUMO}+2$ & -1.27 & 0.20 & 0.25 & 0.12 & 0.43 \\
\hline LUMO+1 & -1.35 & 0.42 & 0.39 & 0.17 & 0.03 \\
\hline LUMO & -2.07 & & & & 1.00 \\
\hline HOMO & -5.50 & 0.66 & & 0.17 & 0.16 \\
\hline HOMO-1 & -6.17 & & & & 1.00 \\
\hline HOMO-2 & -6.53 & 0.12 & & 0.03 & 0.85 \\
\hline HOMO-3 & -6.97 & & & & 1.00 \\
\hline HOMO-4 & -7.73 & 0.01 & 0.47 & 0.03 & 0.48 \\
\hline HOMO-5 & -8.06 & 0.11 & 0.36 & 0.51 & 0.01 \\
\hline HOMO-6 & -8.21 & 0.14 & 0.38 & 0.46 & 0.02 \\
\hline
\end{tabular}


Table S8. Calculated Energies and Components of MOs Near Frontier Orbital Levels for Complex Anion of Re-(OMe) $)_{2}$ bpy

\begin{tabular}{|c|c|c|c|c|c|}
\hline & Energy / eV & $\mathrm{Re}$ & $\mathrm{N}$ & $\mathrm{CN}$ & $(\mathrm{OMe})_{2} \mathrm{bpy}$ \\
\hline LUMO+6 & 0.13 & & & & 1.00 \\
\hline LUMO+5 & 0.12 & 0.29 & 0.11 & 0.51 & 0.10 \\
\hline LUMO+4 & -0.78 & 0.13 & 0.13 & & 0.74 \\
\hline LUMO+3 & -1.23 & 0.20 & 0.21 & 0.04 & 0.55 \\
\hline LUMO+2 & -1.43 & 0.09 & 0.12 & 0.03 & 0.77 \\
\hline LUMO+1 & -1.45 & 0.42 & 0.38 & 0.17 & 0.03 \\
\hline LUMO & -2.30 & 0.02 & & & 0.98 \\
\hline HOMO & -5.69 & 0.71 & & 0.21 & 0.08 \\
\hline HOMO-1 & -7.11 & & & & 1.00 \\
\hline HOMO-2 & -7.36 & & & & 1.00 \\
\hline HOMO-3 & -7.58 & 0.02 & & 0.05 & 0.93 \\
\hline HOMO-4 & -7.90 & 0.03 & 0.50 & 0.03 & 0.44 \\
\hline HOMO-5 & -8.14 & 0.13 & 0.33 & 0.54 & \\
\hline HOMO-6 & -8.29 & 0.15 & 0.35 & 0.48 & 0.02 \\
\hline
\end{tabular}


Table S9. Calculated Energies and Components of MOs Near Frontier Orbital Levels for Complex Anion of Rebpy

\begin{tabular}{cccccc}
\hline & Energy / eV & $\mathrm{Re}$ & $\mathrm{N}$ & $\mathrm{CN}$ & bpy \\
\hline LUMO+6 & 0.06 & 0.32 & 0.09 & 0.50 & 0.09 \\
LUMO+5 & -0.04 & & & 0.03 & 1.00 \\
LUMO+4 & -1.05 & 0.25 & 0.24 & 0.48 \\
LUMO+3 & -1.43 & 0.09 & 0.09 & 0.13 & 0.82 \\
LUMO+2 & -1.57 & 0.44 & 0.38 & 0.04 \\
LUMO+1 & -1.61 & 0.09 & 0.12 & 0.79 \\
LUMO & -2.44 & 0.03 & 0.01 & & 0.96 \\
HOMO & -5.82 & 0.71 & & 0.23 & 0.06 \\
HOMO-1 & -7.10 & & & & 1.00 \\
HOMO-2 & -7.94 & 0.05 & 0.51 & 0.53 & 0.44 \\
HOMO-3 & -8.12 & 0.12 & 0.26 & 0.09 \\
HOMO-4 & -8.31 & 0.15 & 0.35 & 0.11 & 0.02 \\
HOMO-5 & -8.33 & 0.06 & 0.08 & 0.19 & 0.75 \\
HOMO-6 & -8.45 & & & 0.81 \\
\hline
\end{tabular}


Table S10. Calculated Energies and Components of MOs Near Frontier Orbital Levels for Complex Anion of Re-Brabpy

\begin{tabular}{|c|c|c|c|c|c|}
\hline & Energy / eV & $\operatorname{Re}$ & $\mathrm{N}$ & $\mathrm{CN}$ & $\mathrm{Br}_{2} \mathrm{bpy}$ \\
\hline LUMO+6 & -0.54 & & & & 1.00 \\
\hline LUMO+5 & -0.61 & & & & 1.00 \\
\hline LUMO+4 & -1.08 & 0.30 & 0.31 & 0.11 & 0.28 \\
\hline $\mathrm{LUMO}+3$ & -1.52 & 0.42 & 0.38 & 0.17 & 0.03 \\
\hline LUMO+2 & -1.56 & 0.09 & 0.10 & & 0.81 \\
\hline LUMO+1 & -1.76 & 0.03 & 0.04 & & 0.93 \\
\hline LUMO & -2.65 & 0.03 & & & 0.97 \\
\hline HOMO & -5.84 & 0.69 & & 0.21 & 0.10 \\
\hline HOMO-1 & -7.33 & & & & 1.00 \\
\hline HOMO-2 & -7.62 & & & & 1.00 \\
\hline HOMO-3 & -7.76 & & & 0.03 & 0.97 \\
\hline HOMO-4 & -8.02 & 0.04 & 0.49 & 0.03 & 0.44 \\
\hline HOMO-5 & -8.21 & & & & 1.00 \\
\hline HOMO-6 & -8.23 & 0.12 & 0.29 & 0.55 & 0.03 \\
\hline
\end{tabular}


Table S11. Calculated Transition Energies for Complex Anion of Re-(NMe $)_{2} \mathbf{b p y}$

\begin{tabular}{|c|c|c|c|}
\hline $\begin{array}{l}\text { Energy } \\
/ \mathrm{eV}\end{array}$ & $\begin{array}{l}\text { Wave- } \\
\text { length } \\
/ \mathrm{nm}\end{array}$ & $\begin{array}{l}\text { Oscillator } \\
\text { strength }\end{array}$ & $\begin{array}{l}\text { Major MO contribution for the electronic transition } \\
\text { (\% component) }\end{array}$ \\
\hline 2.70 & 459 & 0.0883 & HOMO->LUMO(92), HOMO->L+3(3), HOMO->L+2(2), H-2->LUMO(1) \\
\hline 2.88 & 430 & 0.0027 & HOMO->L+1(97), H-2->L+1(2) \\
\hline 3.11 & 399 & 0.0078 & $\begin{array}{l}\mathrm{HOMO}->\mathrm{L}+2(76), \mathrm{HOMO}->\mathrm{L}+3(13), \mathrm{HOMO}->\mathrm{LUMO}(5), \mathrm{HOMO}->\mathrm{L}+4(4), \\
\mathrm{H}-2->\mathrm{L}+2(1)\end{array}$ \\
\hline 3.45 & 359 & 0.0440 & $\mathrm{H}-1->\mathrm{LUMO}(94), \mathrm{H}-1->\mathrm{L}+2(1)$ \\
\hline 3.68 & 337 & 0.0326 & $\begin{array}{l}\mathrm{HOMO}->\mathrm{L}+3(58), \mathrm{H}-2->\mathrm{LUMO}(21), \mathrm{HOMO}->\mathrm{L}+2(14), \mathrm{HOMO}->\mathrm{L}+4(3), \\
\mathrm{H}-3->\mathrm{LUMO}(2)\end{array}$ \\
\hline 3.79 & 327 & 0.0864 & $\begin{array}{l}\mathrm{H}-2->\mathrm{LUMO}(71), \mathrm{HOMO}->\mathrm{L}+3(11), \mathrm{HOMO}->\mathrm{L}+2(5), \mathrm{HOMO}->\mathrm{L}+4(4), \\
\mathrm{H}-2->\mathrm{L}+3(4), \mathrm{HOMO}->\mathrm{LUMO}(1)\end{array}$ \\
\hline 4.04 & 307 & 0.0291 & $\begin{array}{l}\mathrm{HOMO}->\mathrm{L}+4(86), \mathrm{HOMO}->\mathrm{L}+3(7), \mathrm{H}-3->\mathrm{LUMO}(2), \mathrm{H}-2->\mathrm{L}+2(1), \\
\mathrm{H}-2->\mathrm{LUMO}(1)\end{array}$ \\
\hline 4.09 & 303 & 0.0001 & $\mathrm{H}-1->\mathrm{L}+1(100)$ \\
\hline 4.20 & 295 & 0.0467 & $\mathrm{H}-1->\mathrm{L}+2(84), \mathrm{H}-3->\operatorname{LUMO}(7), \mathrm{H}-1->\mathrm{L}+4(6)$ \\
\hline 4.36 & 284 & 0.0001 & $\mathrm{H}-2->\mathrm{L}+1(97), \mathrm{HOMO}->\mathrm{L}+1(2)$ \\
\hline 4.42 & 281 & 0.0099 & $\mathrm{H}-1->\mathrm{L}+3(94), \mathrm{H}-3->\mathrm{LUMO}(2), \mathrm{H}-2->\mathrm{L}+2(2)$ \\
\hline 4.47 & 277 & 0.0223 & $\begin{array}{l}\mathrm{H}-2->\mathrm{L}+2(84), \mathrm{H}-2->\mathrm{L}+3(6), \mathrm{H}-3->\operatorname{LUMO}(3), \mathrm{H}-3->\mathrm{L}+2(1) \\
\mathrm{H}-1->\mathrm{L}+2(1)\end{array}$ \\
\hline 4.54 & 273 & 0.2176 & $\begin{array}{l}\mathrm{HOMO}->\mathrm{L}+5(27), \mathrm{H}-3->\mathrm{LUMO}(24), \mathrm{HOMO}->\mathrm{L}+6(22), \mathrm{HOMO}->\mathrm{L}+7(13), \\
\mathrm{H}-3->\mathrm{L}+2(3), \mathrm{H}-1->\mathrm{L}+2(2), \mathrm{H}-2->\mathrm{L}+2(1), \mathrm{H}-1->\mathrm{L}+3(1)\end{array}$ \\
\hline 4.55 & 273 & 0.3981 & $\begin{array}{l}\mathrm{H}-3->\mathrm{LUMO}(44), \mathrm{HOMO}->\mathrm{L}+5(13), \mathrm{HOMO}->\mathrm{L}+6(12), \mathrm{H}-3->\mathrm{L}+7(9) \\
\mathrm{H}-1->\mathrm{L}+2(6), \mathrm{H}-2->\mathrm{L}+2(4), \mathrm{H}-1->\mathrm{L}+2(2), \mathrm{H}-1->\mathrm{L}+3(2) \\
\mathrm{H}-1->\mathrm{L}+4(1), \mathrm{HOMO}->\mathrm{L}+3(1), \mathrm{HOMO}->\mathrm{L}+4(1)\end{array}$ \\
\hline 4.67 & 266 & 0.0056 & H-4->LUMO(98), H-4->L+2(1) \\
\hline 4.79 & 259 & 0.0001 & $\mathrm{H}-3->\mathrm{L}+1(99)$ \\
\hline 4.85 & 256 & 0.0151 & $\begin{array}{l}\mathrm{HOMO}->\mathrm{L}+5(39), \mathrm{HOMO}->\mathrm{L}+6(23), \mathrm{HOMO}->\mathrm{L}+9(11), \mathrm{HOMO}->\mathrm{L}+7(11), \\
\mathrm{HOMO}->\mathrm{L}+11(6), \mathrm{H}-2->\mathrm{L}+3(4)\end{array}$ \\
\hline 4.88 & 254 & 0.0981 & $\begin{array}{l}\mathrm{H}-2->\mathrm{L}+3(53), \mathrm{HOMO}->\mathrm{L}+6(9), \mathrm{H}-1->\mathrm{L}+4(8), \mathrm{H}-2->\mathrm{L}+4(7), \\
\mathrm{H}-3->\mathrm{L}+2(6), \mathrm{H}-2->\mathrm{L}+2(3), \mathrm{HOMO}->\mathrm{L}+7(3), \mathrm{HOMO}->\mathrm{L}+5(2), \\
\mathrm{HOMO}->\mathrm{L}+3(1)\end{array}$ \\
\hline 4.96 & 250 & 0.0013 & $\begin{array}{l}\mathrm{HOMO}->\mathrm{L}+7(28), \mathrm{H}-3->\mathrm{L}+2(28), \mathrm{H}-1->\mathrm{L}+4(16), \mathrm{HOMO}->\mathrm{L}+6(15), \\
\mathrm{H}-3->\mathrm{LUMO}(2), \mathrm{H}-3->\mathrm{L}+4(2), \mathrm{H}-7->\mathrm{LUMO}(2), \mathrm{H}-4->\mathrm{L}+1(2)\end{array}$ \\
\hline
\end{tabular}


Table S12. Calculated Transition Energies for Complex Anion of Re-(OMe) $)_{2} \mathbf{b p y}$

\begin{tabular}{|c|c|c|c|}
\hline $\begin{array}{l}\text { Energy } \\
/ \mathrm{eV}\end{array}$ & $\begin{array}{l}\text { Wave- } \\
\text { length } \\
/ \mathrm{nm}\end{array}$ & $\begin{array}{l}\text { Oscillator } \\
\text { strength }\end{array}$ & $\begin{array}{l}\text { Major MO contribution for the electronic transition } \\
\text { (\% component) }\end{array}$ \\
\hline 2.66 & 467 & 0.0772 & HOMO->LUMO(95), HOMO->L+3(3) \\
\hline 2.93 & 424 & 0.0030 & HOMO $->\mathrm{L}+1(99)$ \\
\hline 3.16 & 393 & 0.0040 & $\mathrm{HOMO}->\mathrm{L}+2(54), \mathrm{HOMO}->\mathrm{L}+3(36), \mathrm{HOMO}->\mathrm{L}+4(6), \mathrm{HOMO}->\mathrm{LUMO}(2)$ \\
\hline 3.62 & 343 & 0.0628 & HOMO $->$ L $+3(48), \mathrm{HOMO}->\mathrm{L}+2(42), \mathrm{HOMO}->\mathrm{L}+4(30), \mathrm{H}-1->\mathrm{LUMO}(3)$ \\
\hline 4.00 & 310 & 0.0047 & HOMO $->\mathrm{L}+4(86), \mathrm{HOMO}->\mathrm{L}+3(8), \mathrm{H}-1->\mathrm{LUMO}(4)$ \\
\hline 4.35 & 285 & 0.0462 & $\mathrm{H}-2->\mathrm{LUMO}(85), \mathrm{H}-1->\mathrm{L}+2(10), \mathrm{H}-1->\mathrm{L}+4(2)$ \\
\hline 4.43 & 280 & 0.3955 & $\begin{array}{l}\mathrm{H}-1->\mathrm{LUMO}(67), \mathrm{H}-3->\mathrm{LUMO}(15), \mathrm{H}-1->\mathrm{L}+2(3), \mathrm{HOMO}->\mathrm{L}+3(3), \\
\mathrm{HOMO}->\mathrm{L}+4(2), \mathrm{H}-1->\mathrm{L}+4(1), \mathrm{H}-3->\mathrm{L}+2(1)\end{array}$ \\
\hline 4.58 & 271 & 0.0001 & HOMO->L+5(92), HOMO->L+6(5), H-4->LUMO(1) \\
\hline 4.60 & 270 & 0.0049 & H-4->LUMO(97), HOMO->L+5(1) \\
\hline 4.65 & 267 & 0.1283 & $\begin{array}{l}\mathrm{H}-3->\operatorname{LUMO}(71), \mathrm{H}-1->\operatorname{LUMO}(15), \mathrm{H}-1->\mathrm{L}+3(7), \mathrm{H}-2->\mathrm{L}+2(22), \\
\mathrm{H}-3->\mathrm{L}+3(1)\end{array}$ \\
\hline 4.84 & 256 & 0.0000 & $\mathrm{H}-1->\mathrm{L}+1(100)$ \\
\hline
\end{tabular}


Table S13. Calculated Transition Energies for Complex Anion of Rebpy

\begin{tabular}{|c|c|c|c|}
\hline $\begin{array}{l}\text { Energy } \\
/ \mathrm{eV}\end{array}$ & $\begin{array}{l}\text { Wave- } \\
\text { length } \\
/ \mathrm{nm}\end{array}$ & $\begin{array}{l}\text { Oscillator } \\
\text { strength }\end{array}$ & $\begin{array}{l}\text { Major MO contribution for the electronic transition } \\
\text { (\% component) }\end{array}$ \\
\hline 2.60 & 477 & 0.0622 & HOMO $->$ LUMO(94), HOMO->L+3(3), HOMO->L+1(2) \\
\hline 2.89 & 429 & 0.0040 & $\mathrm{HOMO}->\mathrm{L}+2(99)$ \\
\hline 3.14 & 395 & 0.0135 & HOMO $->\mathrm{L}+1(63), \mathrm{HOMO}->\mathrm{L}+4(16), \mathrm{HOMO}->\mathrm{L}+3(16), \mathrm{HOMO}->\mathrm{LUMO}(4)$ \\
\hline 3.54 & 350 & 0.0272 & HOMO $->\mathrm{L}+3(56), \mathrm{HOMO}->\mathrm{L}+1(30), \mathrm{HOMO}->\mathrm{L}+4(9), \mathrm{H}-1->\mathrm{LUMO}(3)$ \\
\hline 3.79 & 328 & 0.0144 & HOMO $->\mathrm{L}+4(72), \mathrm{HOMO}->\mathrm{L}+3(19), \mathrm{HOMO}->\mathrm{L}+1(4), \mathrm{H}-1->\mathrm{LUMO}(4)$ \\
\hline 4.28 & 290 & 0.4528 & $\begin{array}{l}\mathrm{H}-1->\mathrm{LUMO}(77), \mathrm{H}-1->\mathrm{L}+1(10), \mathrm{HOMO}->\mathrm{L}+3(5), \mathrm{HOMO}->\mathrm{L}+4(1), \\
\mathrm{H}-6->\mathrm{L}+1(1), \mathrm{H}-5->\mathrm{L}+1(1)\end{array}$ \\
\hline 4.47 & 277 & 0.0071 & H-2->LUMO(97), H-2->L+1(1) \\
\hline 4.62 & 268 & 0.0003 & HOMO->L+6(97) \\
\hline 4.71 & 263 & 0.0000 & $\mathrm{H}-1->\mathrm{L}+2(100)$ \\
\hline 4.82 & 257 & 0.0544 & $\begin{array}{l}\mathrm{H}-3->\operatorname{LUMO}(54), \mathrm{H}-1->\mathrm{L}+3(21), \mathrm{H}-1->\mathrm{L}+1(18), \mathrm{H}-1->\operatorname{LUMO}(2), \\
\mathrm{H}-8->\operatorname{LUMO}(1)\end{array}$ \\
\hline 4.86 & 255 & 0.0654 & $\begin{array}{l}\mathrm{H}-3->\operatorname{LUMO}(38), \mathrm{H}-1->\mathrm{L}+1(22), \mathrm{H}-1->\mathrm{L}+3(17), \mathrm{H}-5->\operatorname{LUMO}(13) \\
\mathrm{H}-1->\operatorname{LUMO}(3), \mathrm{H}-8->\operatorname{LUMO}(1), \mathrm{H}-1->\mathrm{L}+4(1)\end{array}$ \\
\hline 4.91 & 252 & 0.0222 & $\begin{array}{l}\mathrm{HOMO}->\mathrm{L}+5(62), \mathrm{H}-1->\mathrm{L}+3(15), \mathrm{H}-1->\mathrm{L}+1(14), \mathrm{H}-6->\operatorname{LUMO}(5), \\
\mathrm{H}-1->\mathrm{LUMO}(2)\end{array}$ \\
\hline
\end{tabular}


Table S14. Calculated Transition Energies for Complex Anion of $\mathbf{R e}-\mathbf{B r} \mathbf{2} \mathbf{b p y}$

\begin{tabular}{|c|c|c|c|}
\hline $\begin{array}{l}\text { Energy } \\
/ \mathrm{eV}\end{array}$ & $\begin{array}{l}\text { Wave- } \\
\text { length } \\
/ \mathrm{nm}\end{array}$ & $\begin{array}{l}\text { Oscillator } \\
\text { strength }\end{array}$ & $\begin{array}{l}\text { Major MO contribution for the electronic transition } \\
\text { (\% component) }\end{array}$ \\
\hline 2.50 & 496 & 0.0855 & HOMO->LUMO(97), HOMO->L+2(2) \\
\hline 3.00 & 413 & 0.0021 & $\mathrm{HOMO}->\mathrm{L}+3(96), \mathrm{HOMO}->\mathrm{L}+2(3)$ \\
\hline 3.14 & 395 & 0.0022 & HOMO $->\mathrm{L}+1(61), \mathrm{HOMO}->\mathrm{L}+2(22), \mathrm{HOMO}->\mathrm{L}+4(15)$ \\
\hline 3.42 & 363 & 0.0539 & $\begin{array}{l}\mathrm{HOMO}->\mathrm{L}+2(43), \mathrm{HOMO}->\mathrm{L}+1(37), \mathrm{HOMO}->\mathrm{L}+4(14), \mathrm{H}-1->\mathrm{LUMO}(3), \\
\mathrm{HOMO}->\mathrm{L}+3(2), \mathrm{HOMO}->\mathrm{LUMO}(1)\end{array}$ \\
\hline 3.71 & 334 & 0.0175 & HOMO $->\mathrm{L}+4(70), \mathrm{HOMO}->\mathrm{L}+2(25), \mathrm{H}-1->\mathrm{LUMO}(2), \mathrm{HOMO}->\mathrm{L}+1(1)$ \\
\hline 4.24 & 292 & 0.2611 & $\begin{array}{l}\mathrm{H}-2->\mathrm{LUMO}(50), \mathrm{H}-1->\operatorname{LUMO}(34), \mathrm{H}-1->\mathrm{L}+1(8), \mathrm{H}-3->\mathrm{LUMO}(3), \\
\mathrm{H}-2->\mathrm{L}+1(1), \mathrm{HOMO}->\mathrm{L}+2(1)\end{array}$ \\
\hline 4.33 & 286 & 0.1954 & $\begin{array}{l}\mathrm{H}-2->\mathrm{LUMO}(41), \mathrm{H}-1->\operatorname{LUMO}(40), \mathrm{H}-3->\operatorname{LUMO}(11), \mathrm{HOMO}->\mathrm{L}+2(2), \\
\mathrm{H}-1->\mathrm{L}+2(2)\end{array}$ \\
\hline 4.38 & 283 & 0.0042 & $\mathrm{H}-4->\mathrm{LUMO}(99)$ \\
\hline 4.46 & 278 & 0.0002 & $\mathrm{HOMO}->\mathrm{L}+5(88), \mathrm{HOMO}->\mathrm{L}+6(9), \mathrm{HOMO}->\mathrm{L}+8(1)$ \\
\hline 4.48 & 277 & 0.0567 & H-3->LUMO(79), H-1->LUMO(14), H-1->L+2(3), H-2->LUMO(1) \\
\hline 4.53 & 274 & 0.0000 & $\mathrm{HOMO}->\mathrm{L}+6(74), \mathrm{HOMO}->\mathrm{L}+8(20), \mathrm{HOMO}->\mathrm{L}+5(6)$ \\
\hline 4.70 & 264 & 0.0003 & $\mathrm{HOMO}->\mathrm{L}+8(78), \mathrm{HOMO}->\mathrm{L}+6(16), \mathrm{HOMO}->\mathrm{L}+5(6)$ \\
\hline 4.74 & 262 & 0.0005 & H-6->LUMO(95), HOMO->L+7(3) \\
\hline 4.74 & 262 & 0.0000 & H-5->LUMO(88), H-7->LUMO(10) \\
\hline 4.75 & 261 & 0.0002 & H-7->LUMO(88), H-5->LUMO(10) \\
\hline 4.78 & 259 & 0.0117 & HOMO->L+7(88), H-1->L+1(4), H-6->LUMO(3), H-1->L+2(2) \\
\hline 4.89 & 254 & 0.0003 & $\mathrm{H}-8->\mathrm{LUMO}(98)$ \\
\hline
\end{tabular}




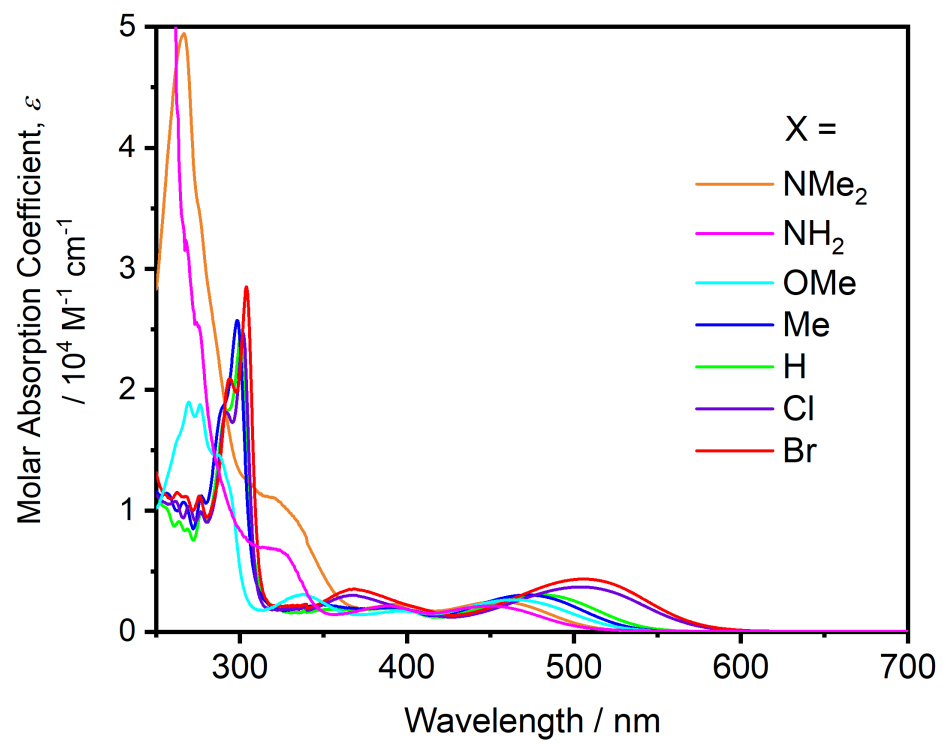

Figure S1. UV-vis spectra of $\mathbf{R e}-\mathbf{X}_{2} \mathbf{b p y}$ [X $=\mathrm{NMe}_{2}$ (orange), $\mathrm{NH}_{2}$ (magenta), $\mathrm{OMe}$ (cyan), $\mathrm{Me}$ (blue), $\mathrm{H}$ (green), $\mathrm{Cl}$ (purple), and $\mathrm{Br}$ (red)] in $\mathrm{EtOH} / \mathrm{MeOH}=4 / 1$ (v/v) at $295 \mathrm{~K}$. 


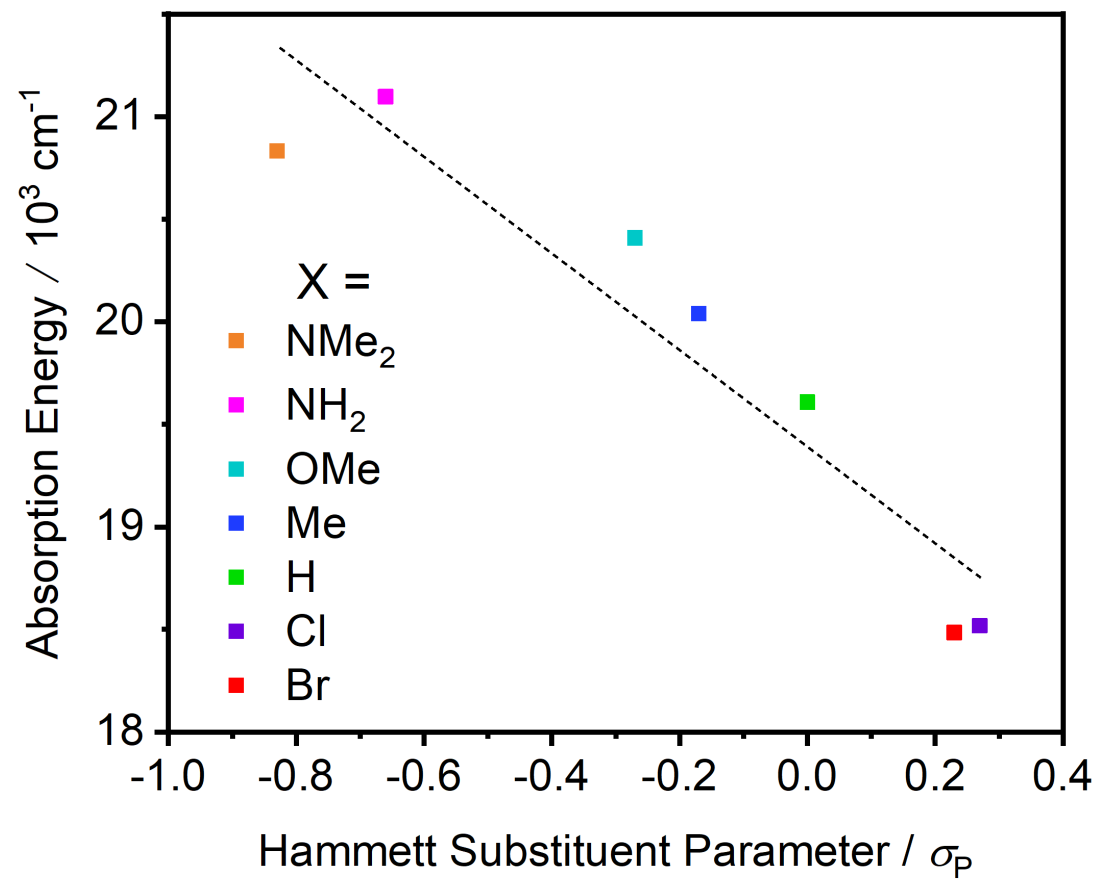

Figure S2. Plot of the absorption energy for $\mathbf{R e}-\mathbf{X}_{2} \mathbf{b p y}$ [X $=\mathrm{NMe}_{2}$ (orange), $\mathrm{NH}_{2}$ (magenta), $\mathrm{OMe}$ (cyan), $\mathrm{Me}$ (blue), $\mathrm{H}$ (green), $\mathrm{Cl}$ (purple), and $\mathrm{Br}$ (red)] as a function of Hammett substituent constant $\left(\sigma_{\mathrm{p}}\right)$. The fit line is drawn by linear least-squares analysis to data points. 


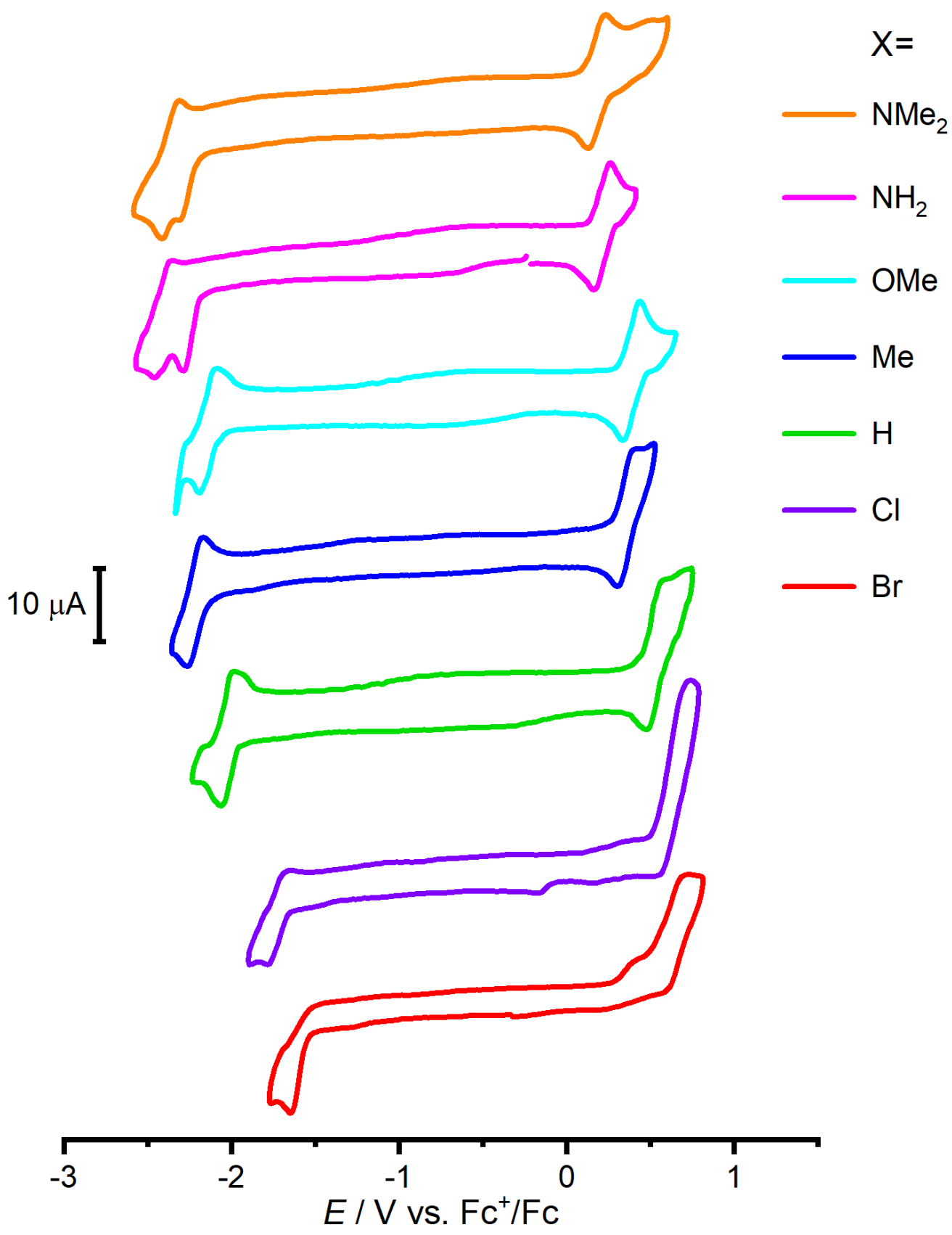

Figure S3. Cyclic voltammograms of $\mathbf{R e}-\mathbf{X}_{2} \mathbf{b p y}\left[\mathrm{X}=\mathrm{NMe}_{2}\right.$ (orange), $\mathrm{NH}_{2}$ (magenta), $\mathrm{OMe}$ (cyan), $\mathrm{Me}$ (blue), $\mathrm{H}$ (green), $\mathrm{Cl}$ (purple), and $\mathrm{Br}$ (red)] in $0.1 \mathrm{M} n-\left(\mathrm{C}_{4} \mathrm{H}_{9}\right)_{4} \mathrm{NPF}_{6}-\mathrm{DMSO}$ at $296 \mathrm{~K}$. 


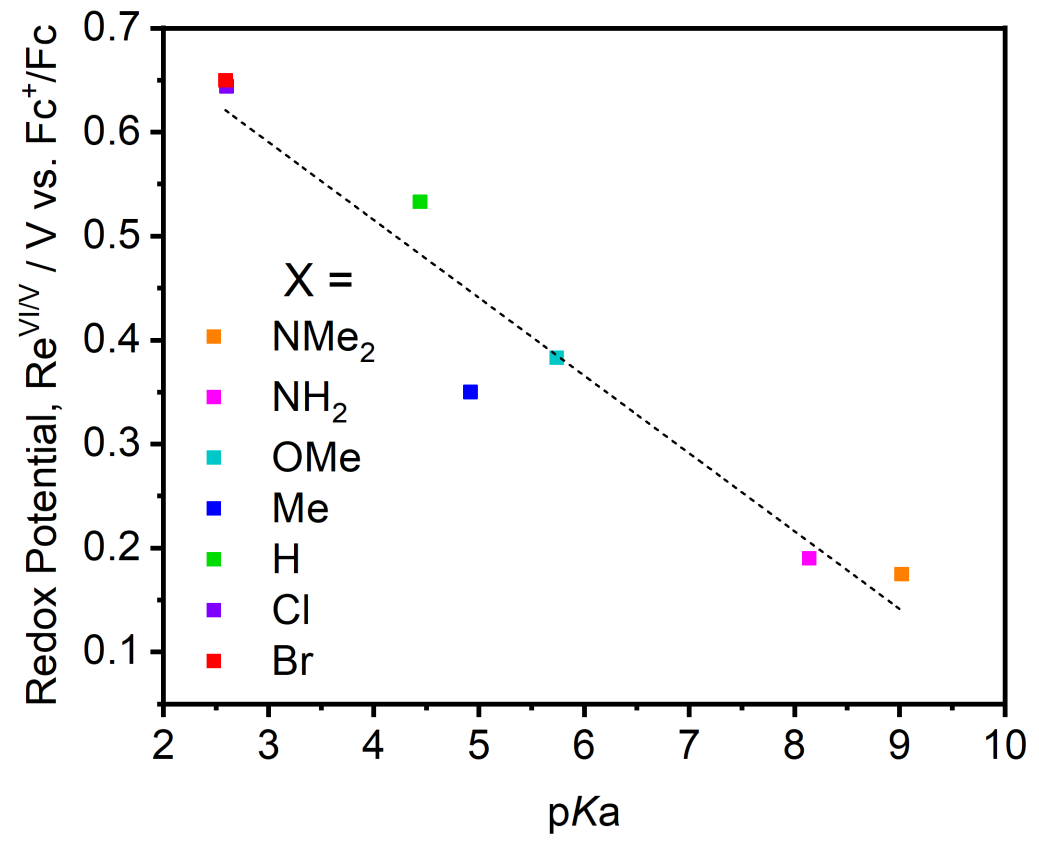

Figure S4. Plot of the $\mathrm{Re}(\mathrm{VI} / \mathrm{V})$ redox potential of $\mathbf{R e}-\mathbf{X}_{2} \mathbf{b p y}\left[\mathrm{X}=\mathrm{NMe}_{2}\right.$ (orange), $\mathrm{NH}_{2}$ (magenta), OMe (cyan), Me (blue), $\mathrm{H}$ (green), $\mathrm{Cl}$ (purple), and $\mathrm{Br}$ (red)] as a function of the calculated $\mathrm{p} K_{\mathrm{a} 1}$ of $\mathrm{X}_{2}$ bpy. The fit line is drawn by linear least-squares analysis to data points. 


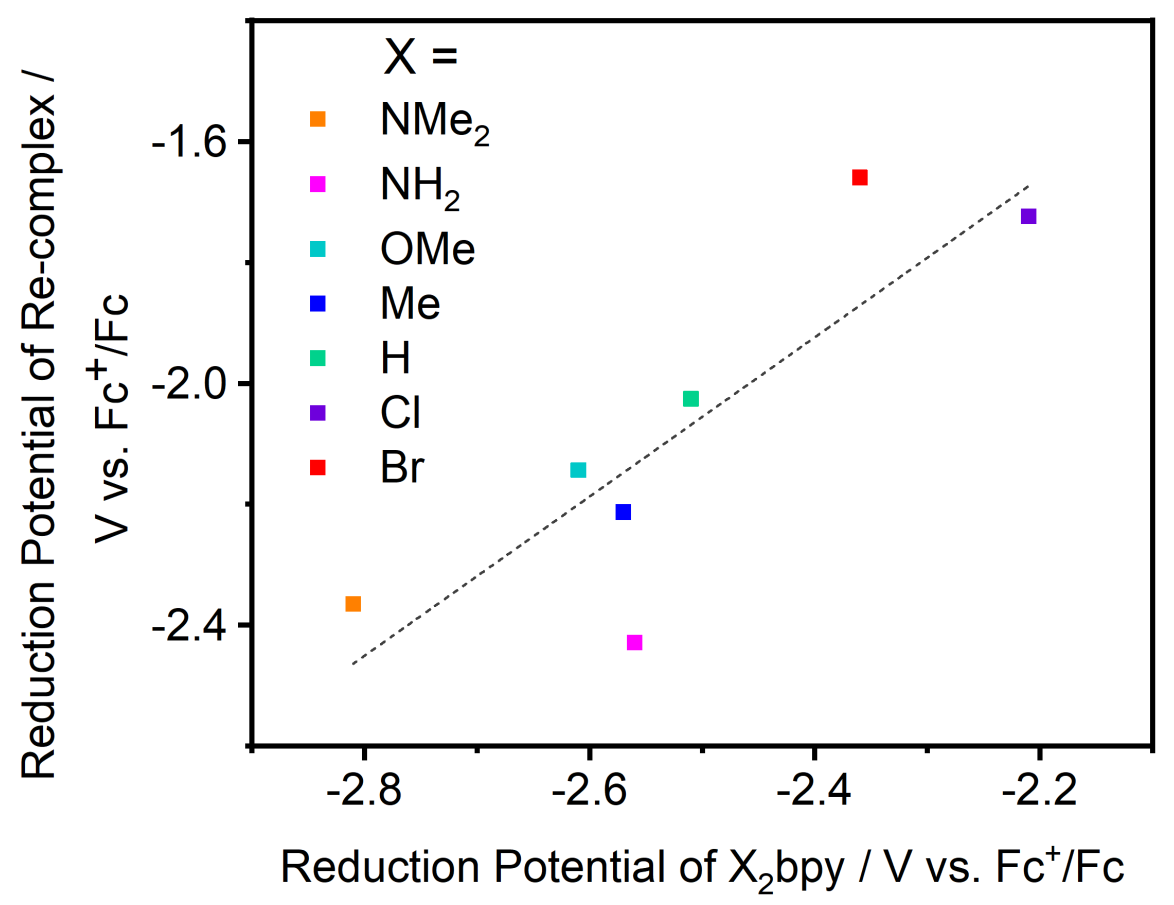

Figure S5. Plot of the reduction potential of $\mathbf{R e}-\mathbf{X}_{2} \mathbf{b p y}\left[\mathrm{X}=\mathrm{NMe}_{2}\right.$ (orange), $\mathrm{NH}_{2}$ (magenta), OMe (cyan), $\mathrm{Me}$ (blue), $\mathrm{H}$ (green), $\mathrm{Cl}$ (purple), and $\mathrm{Br}$ (red)] as a function of reduction potential of $\mathbf{X}_{2} \mathbf{b p y}$. The fit line is drawn by linear least-squares analysis to data points. 


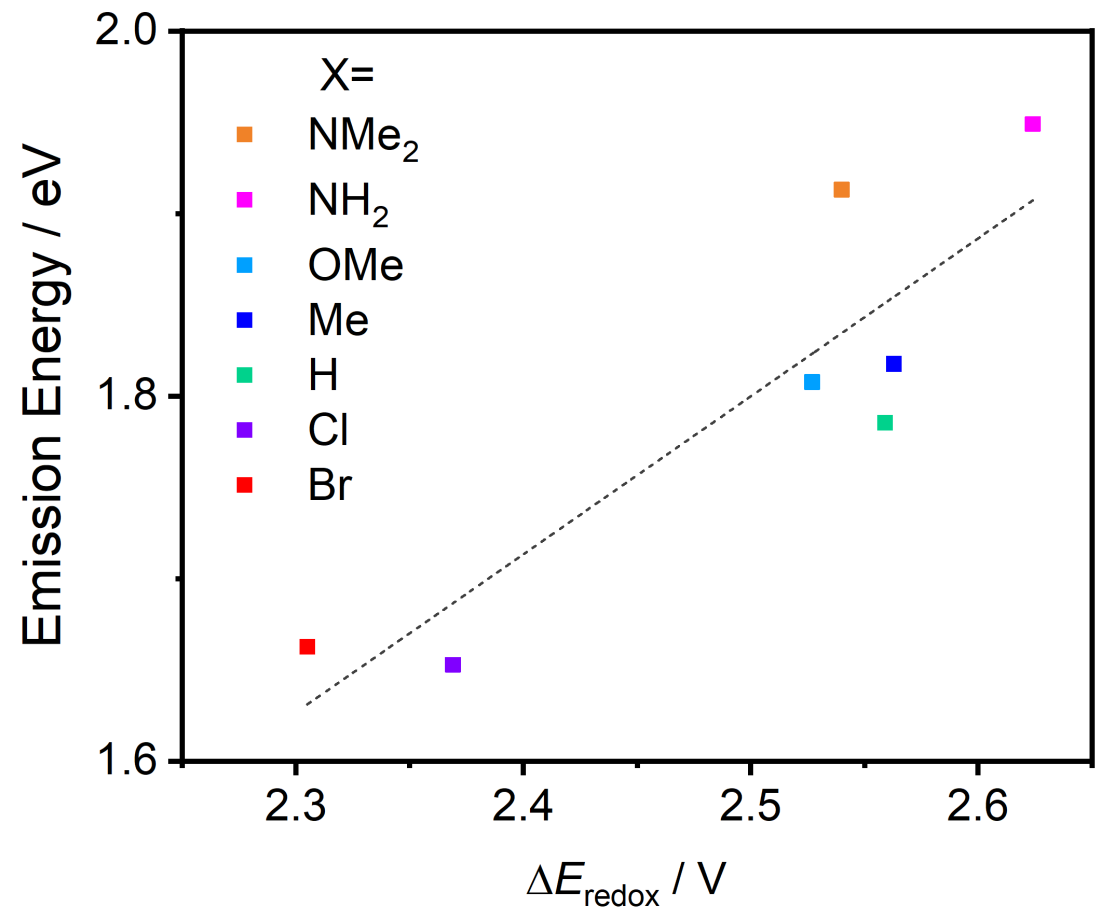

Figure S6. Plot of the emission energy of Re- $\mathbf{X}_{2} \mathbf{b p y}\left[X=\mathrm{NMe}_{2}\right.$ (orange), OMe (cyan), Me (blue), $\mathrm{H}$ (green), $\mathrm{Cl}$ (purple), and $\mathrm{Br}$ (red)] in DMSO at $295 \mathrm{~K}$ as a function of $\Delta E_{\text {redox }}$ in $0.1 \mathrm{M} n-$ $\left(\mathrm{C}_{4} \mathrm{H}_{9}\right)_{4} \mathrm{NPF}_{6}$-DMSO at $296 \mathrm{~K}$. 
(a)

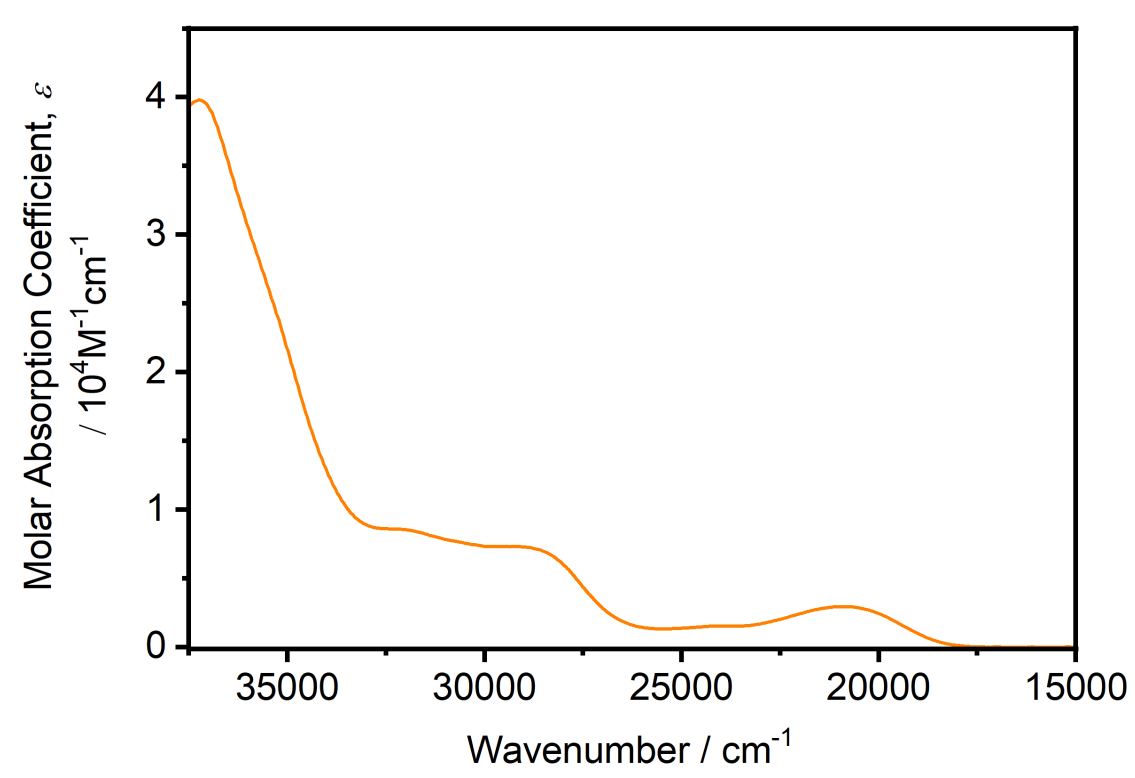

(b)

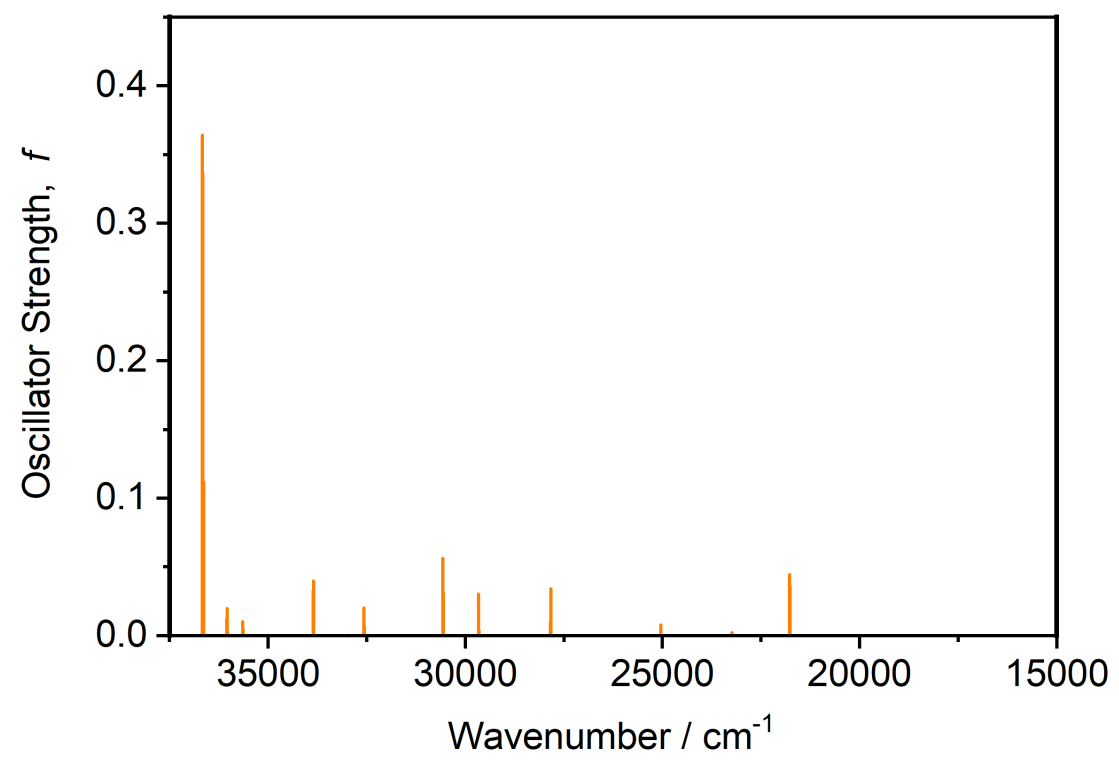

Figure S7. (a) Experimental UV-vis spectrum for Re-(NMe $)_{2} \mathbf{b p y}$ in DMSO and (b) calculated electronic transitions for $\mathbf{R e}-\left(\mathbf{N M e}_{2}\right)_{2} \mathbf{b p y}$ in DMSO. 
(a)

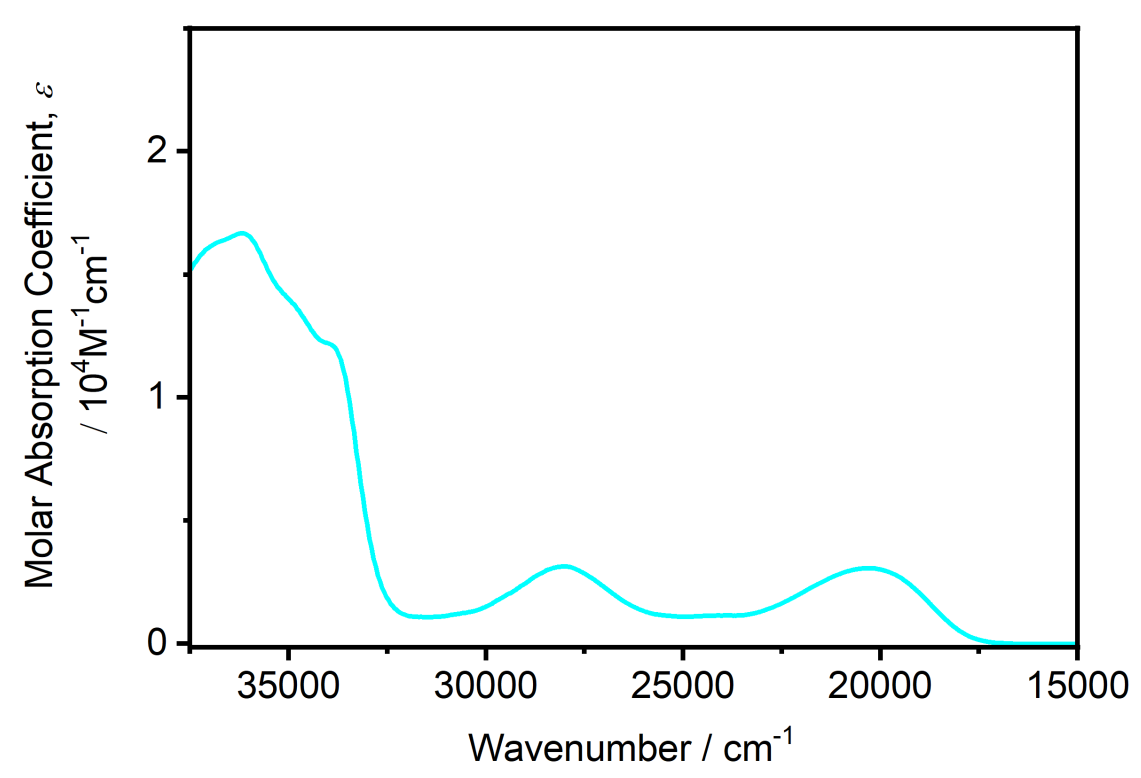

(b)

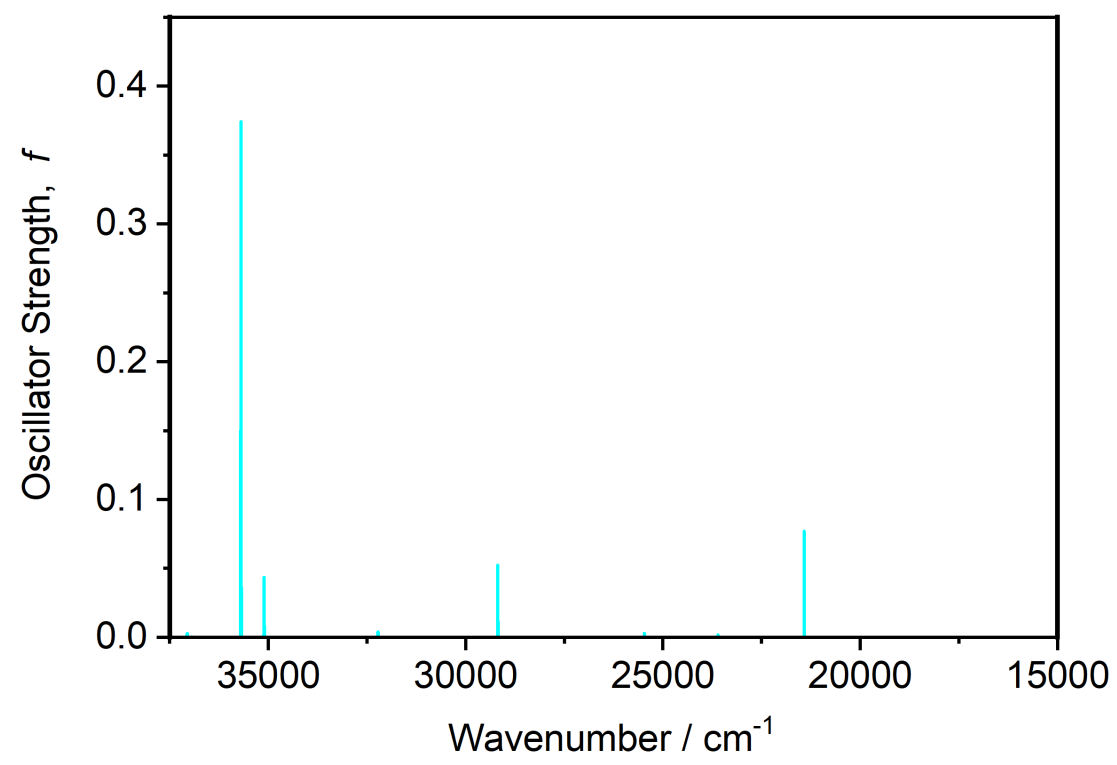

Figure S8. (a) Experimental UV-vis spectrum for Re-(OMe) $)_{2}$ bpy in DMSO and (b) calculated electronic transitions for Re-(OMe $)_{2} \mathbf{b p y}$ in DMSO. 
(a)

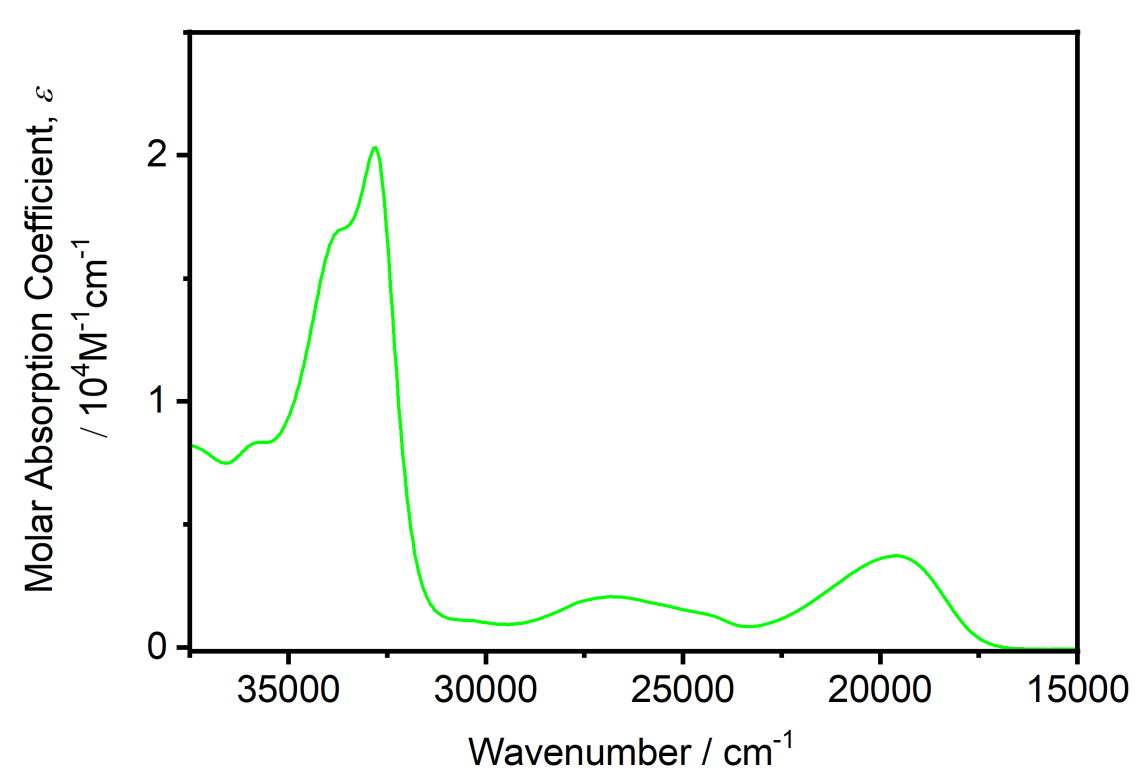

(b)

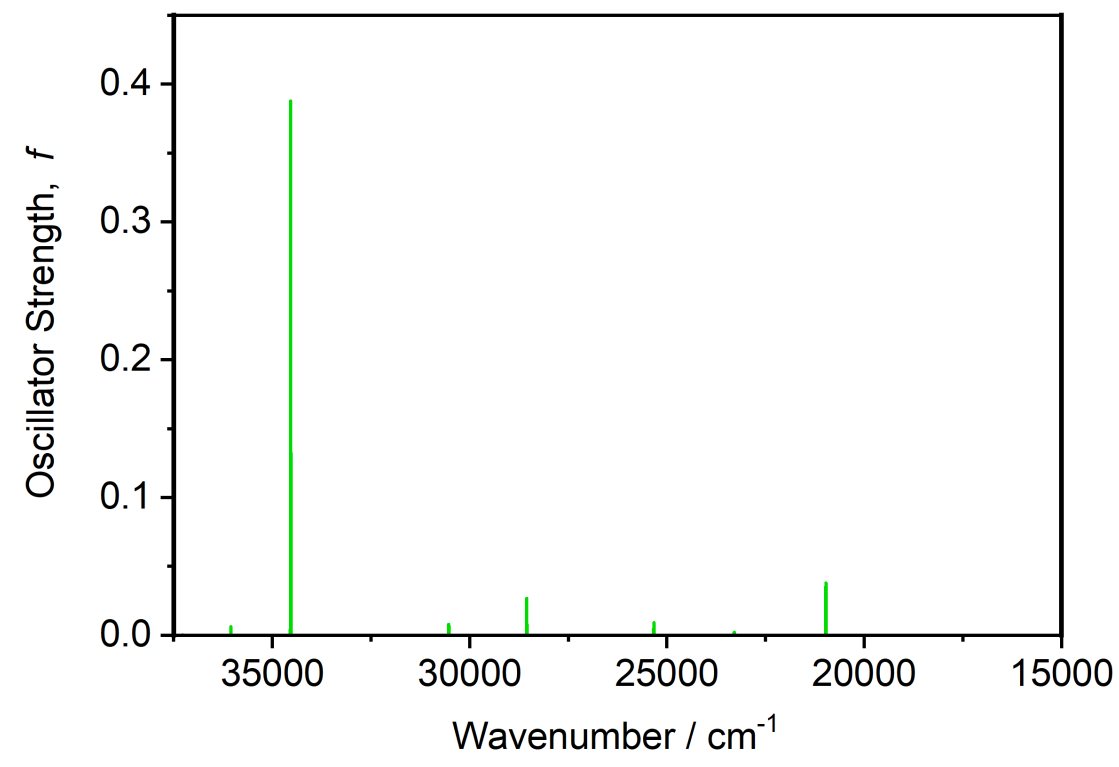

Figure S9. (a) Experimental UV-vis spectrum for Rebpy in DMSO and (b) calculated electronic transitions for Rebpy in DMSO. 
(a)

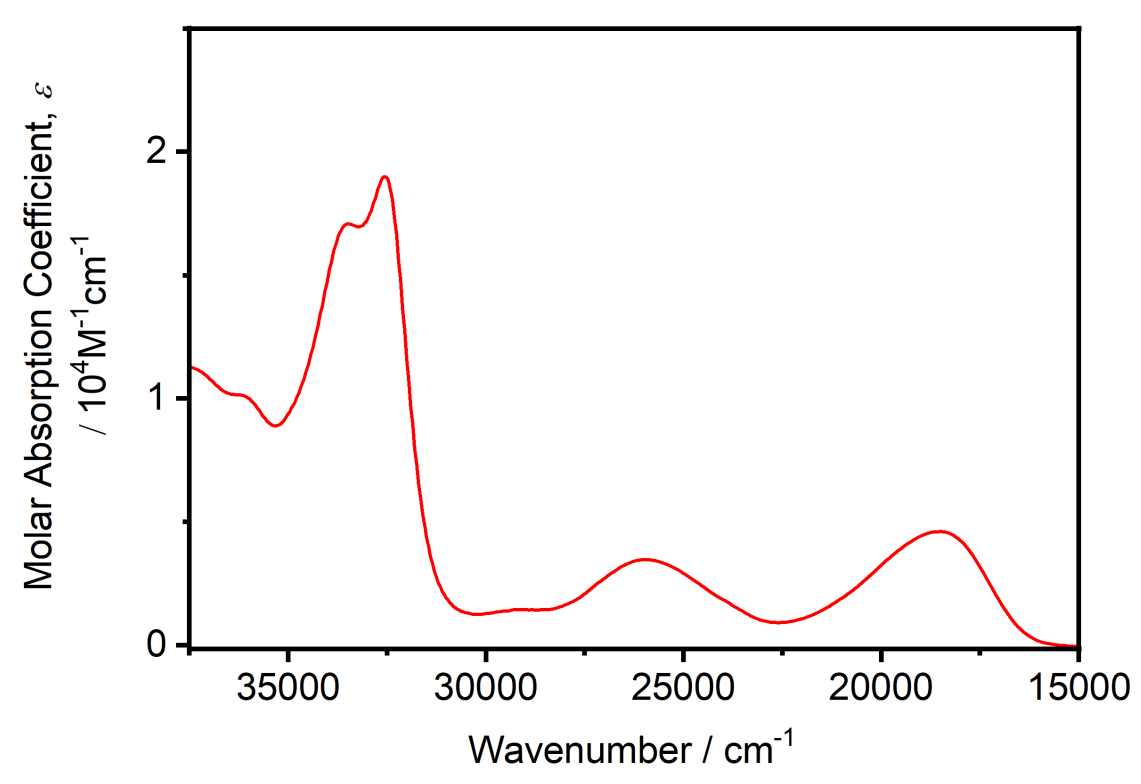

(b)

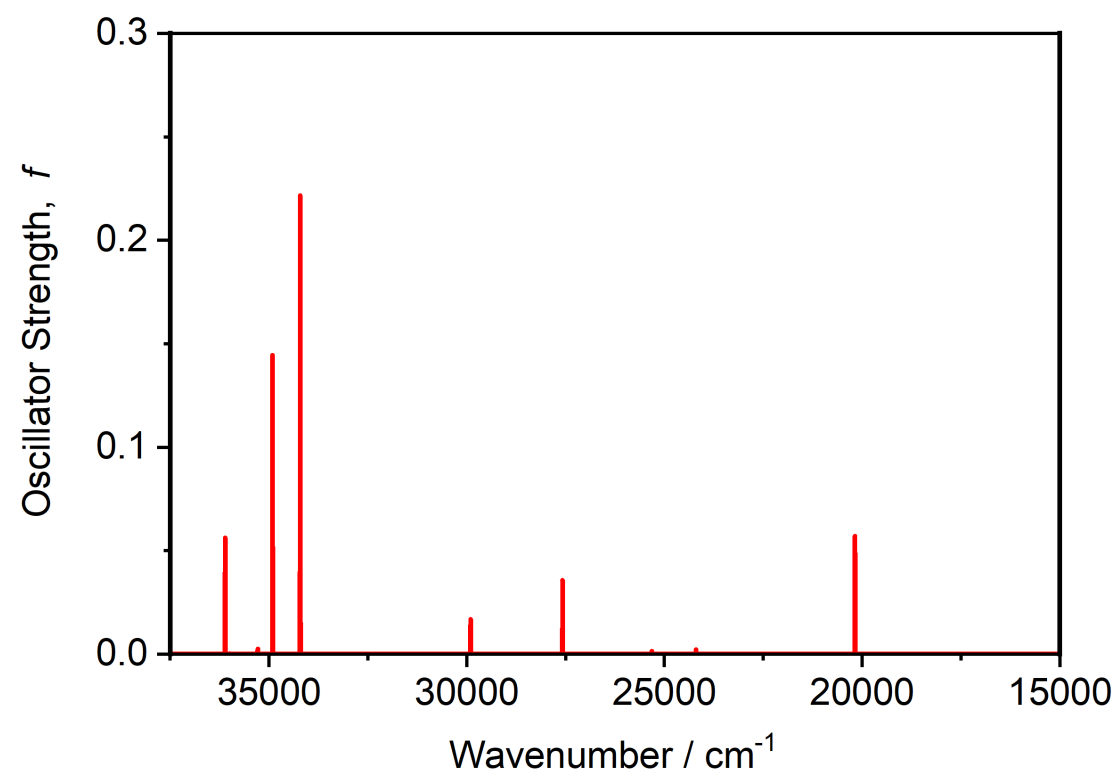

Figure S10. (a) Experimental UV-vis spectrum for $\mathbf{R e}-\mathbf{B r}_{2} \mathbf{b p y}$ in DMSO and (b) calculated electronic transitions for $\mathbf{R e}-\mathbf{B r}_{2} \mathbf{b p y}$ in DMSO. 


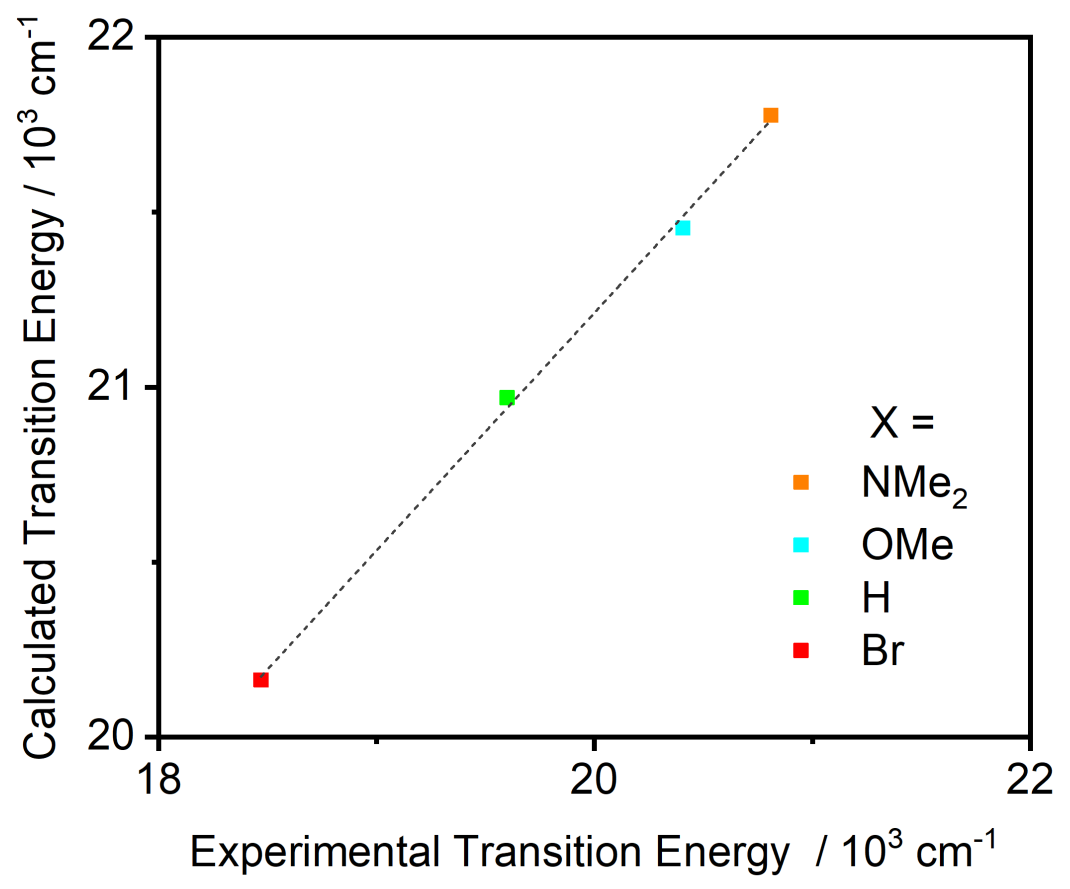

Figure S11. Plot of calculated transition energy as a function of experimental transition energy for $\mathbf{R e}-\mathbf{X}_{2} \mathbf{b p y}$ [X = $\mathrm{NMe}_{2}$ (orange), OMe (cyan), H (green), and $\mathrm{Br}$ (red)] in DMSO. 